\section{Building a Bridge for Batten Disease}

\author{
Melissa L. Feuerborn MDa; Carla C. Keirns MD PhD \\ MSc FACP ${ }^{b}$; Richard J. Barohn MD ${ }^{c}$ \\ a University of Kansas Medical Center, \\ Kansas City, Kansas \\ ${ }^{\mathrm{b}}$ History and Philosophy of Medicine, University \\ of Kansas Medical Center, Kansas City, Kansas \\ c Neuromuscular Medicine, Psychiatry \&. \\ Neurology, University of Missouri, \\ Columbia, Missouri
}

Keywords: Batten, NCL, Rare disease, Advocacy, Caregivers

\section{Introduction}

Living with a rare disease creates unique physical and emotional challenges. It is estimated that nearly 30 million people live with a rare disease in the United States. It is important to realize that a rare disease is not rare for the patient because they often experience the consequences of their disease daily. Fortunately, awareness for rare diseases has grown in recent decades, resulting in advocacy groups for specific rare diseases, organizations for rare diseases in general, and increased research and drug development (1). Exploring the challenges patients experience can help improve the process of diagnosing and raising awareness for rare diseases, while improving the quality of life for individuals with a rare disease.

One of the most common neurodegenerative diseases of childhood is Neuronal Ceroid Lipofuscinosis, or Batten disease (2). It is a group of lysosomal storage disorders linked by the accumulation of an auto fluorescent pigment that leads to progressive degeneration of neuronal tissue (2). To date, fourteen Ceroid Lipofuscinosis, Neuronal genes (abbreviated CLN) have been identified that comprise the NCLs. The exact genetic mutation present determines the clinical manifestations, ultimately leading to the unfortunate outcome of a vegetative state and premature death. The presence of dementia, vision loss, epilepsy, or motor deterioration, especially when present in combination, should raise suspicion for a form of NCL (3). These symptoms can present as early as birth, such as in Congenital CLN1O disease. Neonates are typically born with microcephaly and experience seizures shortly after birth (4). The NCLs can also present as late as adulthood, such as Classic adult onset CLN4 disease. The classic juvenile form caused by a CLN3 gene mutation is thought to be the most prevalent, manifesting between ages 4 and 7 years (5).
Due to the heterogenous nature of the NCLs with multiple dysfunctional protein products, therapeutic targets must be mutation specific to restore the proper metabolic processes. Currently, the only disease modifying agent approved for NCL treatment is Cerliponase alfa, which is a recombinant human proenzyme that replenishes the defective enzyme in CLN2 disease (6). Enzyme replacement therapy and gene therapy are areas of continued exploration in ongoing research. Even though differentiating the NCLs based on the affected gene allows for target treatments to be discovered, it can turn a rare disease into an ultra-rare phenomenon. This dissection can impede the initiation of research and discovery due to a lack of utility. Fortunately, the Batten community is filled with dedicated individuals and organizations working together towards solutions and creating opportunity for growth.

While Batten disease tragically affects children, family members are also impacted by the disease. A 2016 study reported on the experiences of caregivers with children who have inherited metabolic disorders (7). They found that most caregivers established coping strategies, but worried about social challenges for the child. It is essential to recognize the strain a rare and terminal childhood illness can add to families. Fortunately, the emergence of rare disease patient organizations has been incredibly valuable in raising awareness and supporting families. There is a National Rare Disease Day on the last day of February to raise awareness for rare diseases (8). It is difficult for individuals to raise awareness about their disease alone, which can lead to feelings of isolation. Rare disease patient organizations play a vital role in building camaraderie between individuals to help increase funding and researcher interest (9). There are several Batten disease foundations around the world. Additionally, an international conference is held bi-yearly to promote collaboration between researchers working to understand and discover treatments for Batten disease. This project aims to identify areas of difficulty for families affected by Batten disease and what role rare disease organizations play in mitigating these challenges. The areas of interest mainly focus around gaining information pertaining to support and management of the disease and addressing areas of health and wellbeing that are negatively impacted by the disease. By interviewing both caregivers and disease organizations, this study endeavors to analyze the interplay between them.

\section{Methods \\ Participants}

Group 1: Batten Caregivers. We attended the Batten Disease Support and Research Association family meeting, which is an annual event held for families affected by 
Batten disease. We had a table set up where attendees could come get information about the study and volunteer to participate. The interviews were conducted in a private conference room. Interviews were semi-structured with a set of questions relating to the diagnosis process, the impact of the disease on the family, resources available at diagnosis, resources used now, involvement in disease advocacy, and the consequences of being a rare disease. Consent was obtained and responses were audio recorded. Recordings and interview notes are stored on a secure and passwordprotected server. This group consists of seven interviews. Eligible participants for this group are caregivers of individuals diagnosed with Batten disease who volunteered to participate in an interview. Child characteristics provided by caregiver participants are listed in table 1 .

Group 2: Disease Organization Representatives. We identified rare disease organizations involved in Batten disease. Organizations were contacted via email and asked if a representative would be willing to participate in the study. Consent was obtained and interviews were conducted over the phone and audio recorded. Recordings and interview notes are stored on a secure and passwordprotected server. Interviews were semi-structured with a set of questions relating to the role of their organization in the rare disease community, what it means to the interviewee individually, how other people can get involved, and the current state of the rare disease space. This group consists of five interviews. Eligible participants in this group are individuals involved in a rare disease organization. List of organizations interviewed are in table 2.

\section{Results}

\section{Caregiver Interviews}

\section{Identifying Resources after Diagnosis}

Most children in this study were diagnosed through a genetic counselor recommended by a neurologist. Gaining a diagnosis is a vital first step in getting connected to disease organizations. The lifestyle changes that caregivers make to support their child is immense. The in-home accommodations that caregivers reported included features such as a main level bedroom for the child, a supportive chair at the dinner table, and medical equipment utilization. In a child with complex medical needs, it is difficult to find a babysitter. Extended family, often grandparents, played a vital role in providing relief to the parents. Caregivers reported feeling isolated due to a lack of time for social interactions or friendships. Caregivers reported difficulty in providing adequate attention to siblings of their affected child. Caring for a child with Batten disease is multifactorial. Most caregivers reduced the hours they worked or quit working altogether to care for their child.

"After her seventh-gradeyear we brought her home. She started having seizures more frequently... When she ended up that spring with seizures, we were like okay, now it's time for her to be at home. So, we brought her back home and I quit my job that spring, not this year but the spring of last year, May 2017, so that I could be a caregiver for her because we have no other option." (Caregiver)

Fortunately, once connected to the rare disease space, caregivers often found ways of coping. The information about Batten disease that was available to caregivers at the time of diagnosis was variable. Some resources that were identified early included information about the disease, support organizations such as the BDSRA, open clinical trials, and palliative care services. Some caregivers were given information about the most likely diagnoses before the genetic testing results arrived to prepare for a possible diagnosis. Being included in the BDSRA closed Facebook group was advantageous to many caregivers.

"But then Facebook came along. It became easier to keep in touch with other parents and so that's been the biggest thing. I am a part of a couple of different groups specific to Batten, but a lot of it is just being friends and just talking to each other that way... and that's more or less just a place for us to go and vent. It doesn't even have to be about Batten disease. It can be about work or somebody at the store that irritated you or whatever." (Caregiver)

Searching the internet was practical to some caregivers in identifying local resources. When looking for more in-depth data, the amount of material available was intimidating and understanding the information was a common challenge

Table 1. Characteristics of children provided by caregiver participants $(n=7)$

\begin{tabular}{|c|c|c|c|c|c|}
\hline Interview & Type & Gender & Current age & $\begin{array}{l}\text { Symptoms } \\
\text { onset age }\end{array}$ & Diagnosis age \\
\hline $\mathrm{A}$ & CLNl & $\mathrm{M}$ & 8 & 2.5 & 3.5 \\
\hline B & & $\mathrm{F}$ & Deceased & 1 yr., 5 mo. & 2 yr., 2 mo. \\
\hline $\mathrm{C}$ & & $\mathrm{M}$ & 21 & 5.5 & 6.5 \\
\hline $\mathrm{D}$ & CLN2 & $\mathrm{F}$ & 4 & 3 & 3 vr., 9 mo. \\
\hline $\mathrm{E}$ & Unclassified & M & 8 & 2.5 & 8 \\
\hline $\mathrm{F}$ & CLN3 & $\mathrm{F}$ & 14 & 4.5 & 10.5 \\
\hline $\mathrm{G}$ & & $\mathrm{M}$ & 16 & & 10 \\
\hline
\end{tabular}


among caregivers. Reasonably, caregivers looked to the BDSRA to get updates about research, events, and policies related to rare diseases. Many caregivers found conferences helpful to listen and talk with researchers and other families, as one caregiver explained about the family conference.

"It's actually sitting down and talking to somebody who actually knows what they're talking about, that's where the most help has come from. And this past couple of days has been more help than the past 5 years. It's been very beneficial.” (Caregiver)

Many caregivers expressed difficulty in finding reliable information about Batten disease. It is hard to find information that applies specifically to the caregiver's child because of the variability within Batten disease. However, sharing information on the internet has allowed people to expand the knowledge base and as one caregiver stated, "not reinvent the wheel" on how to manage Batten patients. A common trend was that the sooner caregivers were able to connect with other caregivers, the better they were able to navigate managing the disease.

"It's super overwhelming and super emotional, but it's so comforting at the same time to know that I'm not the only one going through this. Where I live, I'm the only one going through this and nobody can possibly understand. But now we've got this extended family that I know I can reach out to and be like I'm frustrated, what do we do from here." (Caregiver)

\section{Addressing Health and Wellbeing}

Caregivers reported that most schools are not well equipped to handle a child with a life limiting disease. There was dissatisfaction in setting appropriate educational goals, which require a balance between promoting learning yet understanding the gradual loss of abilities in the child. One caregiver reported that her daughter attended a school for the blind, which provided tactile based communication devices and more individualized ways of learning. Notably, the BDSRA provides materials to school systems in an effort to guide how they can best serve students with Batten disease.

Children with Batten disease require extensive ancillary services and durable medical equipment (DME) in variable forms and amounts depending on the stage of the disease. Unfortunately, access to these services and equipment can take months and the child's needs may have changed in the interim due to the progression of the disease. Caregivers reported that one way being connected through disease organizations can help with this problem is the ability for them to share DME they are no longer using.
The most common gap in care noted by caregivers was a lack of in-home skilled nursing aid availability. It is incredibly difficult to do everyday tasks such as grocery shop, spend time with siblings, or even get a good night of sleep while caring for a child with complex medical needs. This was especially true for the single parent caregivers who felt like every moment was spent trying to balance life. Even while at work, they would answer phone calls from the school or be thinking about what needs done at home.

"She requires full assist care minus the lifting and the tube feeding. And yet we don't qualify for an in-home aid of any type. And it's like, wow, I mean what do you expect the families to do?" (Caregiver) Connections with other families were crucial in finding an experienced neurologist. It was rare to identify a local neurologist who had managed a Batten disease patient in the past. Caregivers reported that a good neurologist was eager to learn about Batten disease and willing to listen to the caregiver, regardless of whether he/she had experience with Batten disease. Currently, there are three centers of excellence for Batten disease in the United States located in Boston, MA, Columbus, OH, and Rochester, NY. Several caregivers reported visiting one of these centers even if it meant traveling long distances or battling with insurance to cover the visits.

"We were like, okay yes, it's still another two and a half hours. But we know that's a center for excellence for Battens and so we were like, let's go there." (Caregiver)

The biggest challenge reported by caregivers of a child with Batten disease was the emotional toll on the family. Caregivers reported the unbearable reality of seeing their child want to do things that they will never do while losing abilities they used to have. A caregiver revealed the terrifying reality of waking up every morning knowing this could be the last day for her child. Even though this was reported to be the most distressing aspect of caring for a child with Batten disease, having a support group through disease organizations to share these experiences with was an invaluable tool for coping.

\section{Disease Organization Interviews Acting as Connectors}

Most Batten disease specific organizations are focused on connecting families to each other and to resources. Fortunately, Batten families are very supportive to the newly diagnosed in order to help them move forward more smoothly.

"We sit down on the phone with them for an hour, hour and a half, whatever it takes and kind of go over the state of where everything is, what's been 
successful for us, what has not been successful. Just to try to get them on a smoother path than perhaps we had.” (Noah's Hope)

The broader rare disease organizations focused heavily on providing resources to help each disease community make change for themselves and remove the burdens associated with being rare. They connect people to others who have accomplished the same or a similar endeavor. They also have resources such as how to start a 501c3 nonprofit, online courses, webinars, podcasts, drug development education, medical professional education, how to advocate in the community or on the hill, and rare disease summits.

"We are not looking to find a cure for all 7000 rare diseases. We know that that is something that may not be achievable. But what we want to do is not only connect and empower and inspire the rare disease community, but to create a globally connected community equipped to eliminate the challenges of rare disease." (Global Genes)

Leaders in the Batten community took a tragic story and made it one of fortitude and inspiration for others. Most of the Batten organizations are founded by parents of children with Batten disease. This is similar in the general rare disease community. Those involved often had experienced a rare disease in either themselves or someone close to them. After facing the challenges firsthand, people wanted a better future for others.

"I guess I just decided that if I was going to fight that battle and navigate that journey, then I was going to hopefully use my resources and use my education to make it better for families that came behind us." (Rare KC)

\section{Advocacy and Awareness}

The average person may not feel capable of making an impact, but each person can help raise awareness. Disease organization representatives made several suggestions including to reach out to affected families, spread patient stories, share on social media, attend fundraisers, contact the state representatives, educate others that rare diseases are not rare collectively, and be open to learning why it is important to care.

"If you have a child or you personally are going through a cancer, people know it. Because typically you go through chemotherapy, you lose your hair, people know it, they understand it. The problem is with rare disease, it's really hard to define." (Rare $\mathrm{KC})$

Building a sense of camaraderie between individuals with a rare disease is a key role of disease organizations. People are connecting through Facebook, through call or text, and meeting in person at conferences. The BDSRA closed Facebook page was mentioned by most caregivers as especially helpful at the time of first receiving a diagnosis. Having a community to confide in appeared to be invaluable.

"Tragedy brought us together, but we have become good friends and we understand one another. The highs the lows, you really do become a family." (Taylor's Tale)

The representative for Taylor's Tale expressed the importance of advocating for the day-to-day issues that are difficult for the public to understand because these issues are hidden inside the home. Everyday tasks can be challenging. Caregivers try diligently to provide the best quality of life for their child. Advocacy gives people a combined voice where they feel empowered to share the day-to-day issues.

"My heart is in the advocacy part of this because it touches everything." (Taylor's Tale)

Bringing awareness to rare diseases is sometimes done by the media, as pointed out by the representative for the National Organization for Rare Disorders (NORD). Occasionally, a celebrity has a rare disease or has a child with a rare disease. The publicity sheds light on rare diseases while exemplifying that rare can affect anyone. For people who cannot advocate for themselves, rare disease organizations can act as their voice.

\section{Progressing Research}

Many organizations raise money to fund scientific research. When new advancements are made, it creates a legacy for children who have passed from Batten disease. The representative of Noah's Hope explained how he/she attends Batten conferences every year and builds a network that is useful in connecting people to difference resources. $\mathrm{He} /$ she knows the community and can help new scientists move forward, admitting that it is promising to see how Batten research and lysosomal storage disorder research has expanded over the years.

"Every time we see a child that's on ERT (enzyme replacement therapy) now, we can't help but smile knowing that Noah and Laine played a part in making that happen, in changing those children's lives." (Noah's Hope)

In recent years, there has been a large push to involve more patients in the drug development process. Global Genes holds a symposium every year to educate advocates on how to get research moving and find therapies. Rare KC is constantly discussing the topic of research by educating patients in the local community. Topics that are covered include the importance of participation, how to get involved, science terminology, and making connections with researchers. Basic science is important, but many 
organizations are focused on promoting translational science. Taylor's Tale is focused on funding research that can treat patients living with the disease today.

\section{Moving Forward}

Predicting a future for the rare disease community is incredibly optimistic. The representative for Global Genes described the rare disease community as becoming increasingly educated. They connect with other people much faster and create change more efficiently. The NORD representative envisions the future as bright, and a lot of people are working hard to make that true. There is hope that as the new generation replaces the old, they will keep pushing the message of rare disease advocates.

"I think there is a lot of work to do. And that can sometimes feel daunting, but the passion and the drive of the people in this space is so incredible!" (NORD)

Of course, there is still work that needs done for individuals living with a rare disease. Noah's Hope and Taylor's Tale representatives illustrated hurdles the Batten community must work to overcome. The difficulties depend on the stage of the disease. Physically, it is devastating to lose vision, speech, and the ability to move around. Yet importantly, families affected by Batten disease must come to terms with the fact that their future is going to be different than they expected. Even though it is not a desirable destination, many people are working to make it as beautiful of a place as possible.

Notably, the representative for Global Genes shared some insight about ultra-rare disease patients. They may not ever meet someone with their disease and it is more difficult to start research projects. Fortunately, with rare disease organizations individuals are armed with resources to initiate the projects. Therefore, it only takes one person to start making progress for an ultra-rare disease.

"A lot of times these parents that are caring for a chronically ill child, not only do they have to take care of that child, but they are out there raising money and developing registries and finding researchers and learning scientific knowledge that they thought they would never need to learn." (Global Genes)

One of the main areas reported as needing improvement focused on research. Participants described how researchers have made incredible progress with the few funds that are provided for rare diseases. Moreover, industry is playing an increasing role in getting drugs approved for rare diseases. Yet, the rarer forms of Batten are at a roadblock due to a difficulty in partnering with industry.

\section{Discussion}

By interviewing both caregivers and disease organizations, the study was able to analyze the interplay between them. The semi-structured interviews allowed caregivers to elaborate on their experiences and talk about points that they felt were significant. Additionally, the disease organization representatives were able to share personal experiences about their involvement in the organization. Moreover, they could describe their role as a leader in the community and goals for the future.

A key finding from the caregivers in the study was that not having a diagnosis can lead to much distress in caregivers. However, once the diagnosis is made, many families are finding other affected families through social media. The disease organizations are key in providing information about how to be involved in the future of Batten disease. Despite the progress, challenges remain present with regards to education, in-home aid, timely medical equipment, and receiving expert disease management care. Yet through connections with others and support through disease organizations, many of the challenges can be mitigated.

Some key findings from the disease organization representatives in the study were the roles they embodied by acting as connectors for families. The resources they offer are centered around eliminating challenges for families, creating support groups, advocating, and educating. They identify specific problems and work towards solutions, giving a voice to many people who cannot stand alone. The organizations play an active role in progressing research by bringing awareness to specific diseases, funding projects, and encouraging patient participation.

One noteworthy point of bias in the study was that the caregivers were all attendees of the BDSRA family conference. Therefore, they may be more involved in disease organizations than the Batten disease community at large. Additionally, the attendees of the conference had the resources and flexibility to travel. This may represent a group that faces different challenges than people who were not able to attend. It would be beneficial to interview caregivers who are less involved in disease organizations. Another limitation was the small sample size. Each caregiver has a unique story about finding a diagnosis and managing the disease. Added interviews may reveal challenges that were not expressed by caregivers in this study. Despite the mentioned limitations, the study provided valuable information about caregiver experiences and the role of disease organizations. 


\section{Acknowledgements}

Kelly Ranallo and Batten Disease Support \& Research Association (BDSRA) for the caregiver and disease organization connections.

All the interviewees who participated in the study.

\section{Funding}

This work was supported by the Clendening Summer Fellowship through the Department of History and Philosophy of Medicine at the University of Kansas Medical Center.

This work was supported by a CTSA grant from NCATS awarded to the University of Kansas for Frontiers: University of Kansas Clinical and Translational Science Institute (\# UL1TR002366) The contents are solely the responsibility of the authors and do not necessarily represent the official views of the NIH or NCATS.

Declarations of interest: none

\section{Corresponding Author}

Melissa Feuerborn

233 N Main, 217

Salt Lake City, UT 84103

melissa.feuerborn@hsc.utah.edu

\section{References}

1. Griggs R, Batshaw M, Dunkle M, et al. Clinical research for rare disease: Opportunities, challenges, and solutions. Molecular Genetics and Metabolism. 2009 Jan;96(1):20-6. DOI:10.1016/j.ymgme.2008.10.003
2. Nita D, Mole S, Minassian B. Neuronal ceroid lipofuscinoses. Epileptic Disorders. 2016 Sep 1;18(S2):73-88. DOI:10.11684/epd.2016.0844

3. Schulza A, Kohlschüttera A, Minkb J, Simonatic A, Williamsd R. NCL diseases - clinical perspectives. Biochim Biophys Acta. 2013 Nov;1832(11):1801-1806. DOI:10.1016/j.bbadis.2013.04.008

4. Barohn R, Dowd D, Kagan-Hallet K. Congenital Ceroid-Lipofuscinosis. Pediatric Neurology. Jan-Feb 1992;8(1):54-9. DOI:10.1016/0887-8994(92)90054-3

5. Freund K, Sarraf D, Mieler W, Yannuzzi L. The Retinal Atlas. Elsevier Inc. 2017;3:233-278.

6. Kohlschütter A, Schulz A, Bartsch U, Storch S. Current and Emerging Treatment Strategies for Neuronal Ceroid Lipofuscinoses. CNS Drugs. 2019 Apr;33(4):315325. DOI:10.1007/s40263-019-00620-8

7. Siddiq S, Wilson B, Graham I. Experiences of caregivers of children with inherited metabolic diseases: a qualitative study. Orphanet Journal of Rare Diseases. 2016 Dec 7;11(1):168. DOI:10.1186/s13023-016-0548-2

8. Rarediseaseday.org [Internet]. Eurordis Rare Diseases Europe. c2020. Available from: https://www. rarediseaseday.org/article/what-is-rare-disease-day

9. Pinto D, Martin D, Chenhall R. The involvement of patient organisations in rare disease research: a mixed methods study in Australia. Orphanet Journal of Rare Diseases. 2016 Jan 12;11:2. DOI:10.1186/s13023-0160382-6 